\title{
THE INITIAL JUMPS OF SOLUTIONS AND INTEGRAL TERMS IN SINGULAR BVP OF LINEAR HIGHER ORDER INTEGRO-DIFFERENTIAL EQUATIONS
}

\author{
M.K. DAUYLBAYEV AND N. ATAKHAN
}

Received 20 November, 2014

\begin{abstract}
We consider the two-point boundary value problem for the singularly perturbed higher order linear integro-differential equation. The initial jumps of solutions and integral terms are proved. The constructive formulae and asymptotic estimations for solutions and their derivatives are obtained.
\end{abstract}

2010 Mathematics Subject Classification: 34B10; 34B15

Keywords: singularly perturbation, integro-differential equation, small parameter, initial jump, asymptotic method, asymptotic expansion, boundary layer

\section{INTRODUCTION}

Interests to singularly perturbed equations are due to the fact that they are mathematical models of many applied problems associated with processes of diffusion, heat and mass transfers, chemical kinetics and combustion, problems of heat propagation in subtle bodies, theory of semiconductors, dynamics of gyroscopes, quantum mechanics, biology and biophysics, and many others. Various asymptotic methods exist to approximate solutions of certain singularly perturbed problems. Investigations by Lyusternik, Vishik, Vasil'eva and Butuzov [21,22, 24], led to the development the method of boundary functions. This method is also known as the method of boundary layer corrections (O'Malley [14], [15], Smith [2]). Let us name some other asymptotic methods: matching of asymptotic expansions (Kevorkian and Cole [8], Lagerstrom [9], Eckhaus [2]), matching applied to relaxation oscillations (Mischenko and Rozov [11], Grasman [4]), WKB-method and matching for turning point problems (Fedoriuk [3], Olver [13]), multiple scales techniques (Kevorkian and Cole [8], Kapila [5]), averaging method (Bogoliubov and Mitropolskii [1], Sanders and Verhulst [19]), regularization of singularly perturbed problems (Lomov [10]).

Development of different asymptotic methods can be found, e.g., in O'Malley [15] and Vasil'eva [20].

For a nonlinear ordinary differential equation of the second order with a small parameter the initial value problem with singular initial conditions was studied by 
M. I. Vishik and L. A. Lyusternik [23] and K. A. Kasymov [6]. They show that the solution of the original initial value problem tends to the solution of the degenerate equation with modified initial conditions, when the small parameter approaches zero. Such problems became known as the Cauchy problems with initial jumps. The most general cases of the Cauchy problem for singularly perturbed nonlinear systems of ordinary differential and integro-differential equations, as well as for partial differential equations of hyperbolic type were studied by K. A. Kasymov [7]. Boundary problems for singularly perturbed ordinary differential equations of higher order with the initial jump was considered in [12].

In this work we consider boundary value problems for singularly perturbed $\mathrm{n}$-th order linear integro-differential equations, when fast variables of solution $y^{(i)}(t, \varepsilon), i=$ $\overline{m_{1}+1, n-1}$ satisfy $y_{0}^{i}(\varepsilon) \rightarrow \infty$ as $\varepsilon \rightarrow 0$ near to the boundary of the domain. It is shown that there is the phenomenon of initial jumps not only of solutions, but also of the integral terms. The boundary-value problems were investigated in [16], [17] by using parametrization and the numerical analytic method based upon successive approximations [18].

\section{STATEMENT OF THE PROBLEM AND PRELIMINARY MATERIALS}

Consider the following integro-differential equation

$$
L_{\varepsilon} y \equiv \varepsilon y^{(n)}+A_{1}(t) y^{(n-1)}+\ldots+A_{n}(t) y=F(t)+\int_{0}^{1} \sum_{i=0}^{m_{1}+1} H_{i}(t, x) y^{(i)}(x) d x
$$

with boundary conditions

$$
h_{i} y(t, \varepsilon) \equiv \sum_{j=0}^{m_{i}} \alpha_{i j} y^{(j)}(0, \varepsilon)+\sum_{j=0}^{l_{i}} \beta_{i j} y^{(j)}(1, \varepsilon)=a_{i}, \quad i=\overline{1, n}
$$

where $\varepsilon>0$ is small parameter, $\alpha_{i j}, \beta_{i j}, a_{i} \in R$ are known constants independent of $\varepsilon$.

Assume that the following conditions hold:

C1) $A_{i}(t), F(t), i=\overline{1, n}$, are sufficiently smooth functions defined on the interval $[0,1]$

C2) $A_{1}(t) \geq \gamma=$ const $>0,0 \leq t \leq 1$;

C3) $\alpha_{1, m_{1}} \neq 0, n-1>m_{1}>m_{2} \geq \ldots \geq m_{n}, n-1>l_{1}>l_{2} \geq \ldots \geq l_{n}$, where $m_{i}, l_{i}$ are nonnegative integers; 
C4) $\bar{\Delta}=\left|\begin{array}{ccc}h_{2} y_{10}(t) & \ldots & h_{2} y_{n-1,0}(t) \\ \ldots & \ldots & \ldots \\ h_{n} y_{10}(t) & \ldots & h_{n} y_{n-1,0}(t)\end{array}\right| \neq 0$,

where $y_{i 0}(t), i=1, \ldots, n-1$, is the fundamental set of solutions of the following homogeneous degenerate differential equation

$$
L_{0} y_{0}(t) \equiv A_{1}(t) y_{0}^{(n-1)}+\ldots+A_{n}(t) y_{0}(t)=0
$$

C5) Functions $H_{i}(t, x), i=0, \ldots, m_{1}+1$, are defined in the domain $D=(0 \leq$ $t \leq 1,0 \leq x \leq 1)$ and sufficiently smooth.

Some other conditions will be imposed later. We consider the following homogeneous singularly perturbed equation associated with (2.1)

$$
L_{\varepsilon} y \equiv \varepsilon y^{(n)}+A_{1}(t) y^{(n-1)}+\ldots+A_{n}(t) y=0 .
$$

Lemma 1. If conditions $(C 1)$ and $(C 2)$ are satisfied, then the fundamental set of solutions $y_{i}(t, \varepsilon), i=1, \ldots, n$, of (2.4) in the interval $0 \leq t \leq 1$, has the following asymptotic representation as $\varepsilon \rightarrow 0$.:

$$
\left\{\begin{array}{l}
y_{i}^{(j)}(t, \varepsilon)=y_{i 0}^{(j)}(t)+O(\varepsilon), i=1, \ldots, n-1, j=0, \ldots, n-1, \\
y_{n}^{(j)}(t, \varepsilon)=\frac{1}{\varepsilon^{j}} \exp \left(\frac{1}{\varepsilon} \int_{0}^{t} \mu(x) d x\right)\left(\mu^{j}(t) y_{n 0}(t)+O(\varepsilon)\right), j=0, \ldots, n-1,
\end{array}\right.
$$

where $\mu(t)=-A_{1}(t)<0, y_{i 0}(t), i=1, \ldots, n-1$, are solutions of the problem

$$
\begin{gathered}
L_{0} y_{0} \equiv A_{1}(t) y_{0}^{(n-1)}(t)+\ldots+A_{n}(t) y_{0}(t)=0, \\
y_{i 0}^{(j)}(0)=\left\{\begin{array}{ll}
1, & j=i-1, \\
0, & j \neq i-1,
\end{array} j=0, \ldots, n-2, i=1, \ldots, n-1,\right.
\end{gathered}
$$

and

$$
y_{n 0}(t)=\left(A_{1}(0) / A_{1}(t)\right)^{n-1} \exp \left(\int_{0}^{t}\left(A_{2}(x) / A_{1}(t)\right) d x\right) .
$$

The proof of the Lemma 1 is readily obtained from the known theorems of L. Schlesinger, G. D. Birkhoff, and P. Noaillon [10].

Definition 1. Let the function $K(t, s, \varepsilon), 0 \leq s \leq t \leq 1$, be a solution for the following problem

$$
L_{\varepsilon} K(t, s, \varepsilon)=0, K^{(j)}(s, s, \varepsilon)=0, j=0, \ldots, n-2, K^{(n-1)}(s, s, \varepsilon)=1 .
$$


The Cauchy function, $K(t, s, \varepsilon)$, can be represented as

$$
K(t, s, \varepsilon)=\frac{W_{n}(t, s, \varepsilon)}{W(s, \varepsilon)},
$$

where $W(s, \varepsilon)$ is the Wronskian of the fundamental set of solutions $y_{1}(s, \varepsilon), \ldots$, $y_{n}(s, \varepsilon)$ of equation $(2.4), W_{n}(t, s, \varepsilon)$ is the $\mathrm{n}$-th order determinant obtained from the Wronskian $W(s, \varepsilon)$ by replacing the n-th row with the fundamental set of solutions $y_{1}(t, \varepsilon), \ldots, y_{n}(t, \varepsilon)$.

Lemma 2. If conditions $C 1)$ and $C 2$ ) are satisfied, then for sufficiently small $\varepsilon$ the Cauchy function $K(t, s, \varepsilon)$ in the domain $0 \leq s \leq t \leq 1$ can be represented as followings

$$
\begin{gathered}
K^{(j)}(t, s, \varepsilon)=-\varepsilon \frac{\bar{W}_{n-1}^{(j)}(t, s)}{\mu(s) \bar{W}(s)}+\varepsilon^{n-1-j} \exp \left(\frac{1}{\varepsilon} \int_{s}^{t} \mu(x) d x\right) \times \\
\times \frac{\mu^{j}(t) y_{n 0}(t)}{\mu^{n-1}(s) y_{n 0}(s)}+O\left(\varepsilon^{2}+\varepsilon^{n-j} \exp \left(\frac{1}{\varepsilon} \int_{s}^{t} \mu(x) d x\right)\right),
\end{gathered}
$$

where $y_{n 0}(t)$ is given by (2.6), the determinant $\bar{W}(s)$ is the Wronskian of the fundamental set of solutions $y_{10}(s), \ldots, y_{n-1,0}(s)$ of equation $(2.3)$, and $\bar{W}_{n-1}^{(j)}(t, s)$ is the determinant obtained from $\bar{W}(s)$ by replacing the $(n-1)$-th row with $y_{10}(t), \ldots$, $y_{n-1,0}(t)$.

The proof of the Lemma 2 immediately follows by means of (2.5) and (2.8).

Definition 2. The functions $\Phi_{i}(t, \varepsilon), i=1, \ldots, n$, are called boundary functions for the boundary value problem (2.1) and (2.2), if they satisfy the following problem

$$
L_{\varepsilon} \Phi_{i}(t, \varepsilon)=0, i=1, \ldots, n, h_{k} \Phi_{i}(t, \varepsilon)= \begin{cases}1, & i=k, \\ 0, & i \neq k, k=1, \ldots, n .\end{cases}
$$

Let us consider the determinant

$$
\Delta(\varepsilon)=\left|\begin{array}{ccc}
h_{1} y_{1}(t, \varepsilon) & \ldots & h_{1} y_{n}(t, \varepsilon) \\
\ldots & \ldots & \ldots \\
h_{n} y_{1}(t, \varepsilon) & \ldots & h_{n} y_{n}(t, \varepsilon)
\end{array}\right| .
$$

For the determinant $\Delta(\varepsilon)$ using formulas (2.2) and (2.5), we obtain the following asymptotic representation as $\varepsilon \rightarrow 0$

$$
\Delta(\varepsilon)=(-1)^{1+n} \frac{\mu^{m_{1}}(0)}{\varepsilon^{m_{1}}}\left(\alpha_{1, m_{1}} \bar{\Delta}+O(\varepsilon)\right),
$$

where $\bar{\Delta}$ is the determinant given by the condition $\mathrm{C} 4$. 
The boundary functions $\Phi_{i}(t, \varepsilon), \quad i=1, \ldots, n$, on the interval $[0,1]$ can be represented in the form

$$
\Phi_{i}(t, \varepsilon)=\frac{\bar{\Delta}_{i}(t, \varepsilon)}{\Delta(\varepsilon)}, \quad i=1, \ldots, n,
$$

where $\Delta_{i}(t, \varepsilon)$ is the determinant obtained from $\Delta(\varepsilon)$ by replacing the $i$-th row by the fundamental set of solutions $y_{1}(t, \varepsilon), \ldots, y_{n}(t, \varepsilon)$ of the equation (2.4).

Lemma 3. If conditions $(C 1)-(C 4)$ are satisfied, then for boundary functions $\Phi_{i}(t, \varepsilon), \quad i=1, \ldots, n$, in the interval $0 \leq t \leq 1$ we obtain the following asymptotic representation as $\varepsilon \rightarrow 0$

$$
\begin{aligned}
& \Phi_{1}^{(j)}(t, \varepsilon) \\
& =-\varepsilon^{m_{1}-m_{2}} \frac{\alpha_{2, m_{2}} \bar{\Delta}_{1}^{(j)}(t)}{\mu^{m_{1}-m_{2}}(0) \alpha_{1, m_{1}} \bar{\Delta}}+\varepsilon^{m_{1}-j} \cdot \frac{y_{n 0}(t) \mu^{j}(t)}{\mu^{m_{1}}(0) \alpha_{1, m_{1}}} \exp \left(\frac{1}{\varepsilon} \int_{0}^{t} \mu(x) d x\right) \\
& +O\left(\varepsilon^{m_{1}-m_{2}+1}+\varepsilon^{m_{1}+1-j} \exp \left(\frac{1}{\varepsilon} \int_{0}^{t} \mu(x) d x\right)\right), \quad j=0, \ldots, n-1, \\
& \Phi_{i}^{(j)}(t, \varepsilon)=\frac{\bar{\Delta}_{i-1}^{j}(t)}{\bar{\Delta}}+(-1)^{i-1} \varepsilon^{m_{1}-j} \cdot \frac{y_{n 0}(t) \mu^{j}(t) \bar{\Delta}_{i}}{\mu^{m_{1}}(0) \alpha_{1, m_{1}} \bar{\Delta}} \times \\
& \times \exp \left(\frac{1}{\varepsilon} \int_{0}^{t} \mu(x) d x+O\left(\varepsilon+\varepsilon^{m_{1}+1-j} \exp \left(\frac{1}{\varepsilon} \int_{0}^{t} \mu(x) d x\right)\right)\right), \\
& j=0, \ldots, n-1 ; i=2, \ldots, n,
\end{aligned}
$$

where $\bar{\Delta}_{i}^{(j)}(t)$ is the determinant obtained from $\bar{\Delta}$ by replacing the $i$-th row with the fundamental set of solutions $y_{10}^{(j)}(t), \ldots, y_{n-1,0}^{(j)}(t)$ of the equation (2.3), and $\bar{\Delta}_{i}, \quad i=2, \ldots, n$, are determinants obtained from the matrix

$$
\left(\begin{array}{ccc}
h_{1} y_{10}(t) & \ldots & h_{1} y_{n-1,0}(t) \\
\ldots & \ldots & \ldots \\
h_{n} y_{10}(t) & \ldots & h_{n} y_{n-1,0}(t)
\end{array}\right)
$$

after deleting the $i$-th row.

The proof of the Lemma 3 follows from (2.12) and by taking into account (2.2), (2.5), and (2.11). 


\section{MAIN RESULTS}

We seek to find the solution of the boundary value problem (2.1) and (2.2) in the form

$$
y(t, \varepsilon)=C_{1} \Phi_{1}(t, \varepsilon)+\ldots+C_{n} \Phi_{n}(t, \varepsilon)+\frac{1}{\varepsilon} \int_{0}^{t} K(t, s, \varepsilon) z(s, \varepsilon) d s,
$$

where $K(t, s, \varepsilon)$ is the Cauchy function, $\Phi_{i}(t, \varepsilon), \quad i=1, \ldots, n$, are the boundary functions, $C_{i}, i=1, \ldots, n$, are unknown constants, and $z(s, \varepsilon)$ is an unknown function. Use (3.1) in (2.1), to obtain that $z(s, \varepsilon)$ satisfies the following Fredholm integral equation of the second kind

$$
z(s, \varepsilon)=F(t)+\sum_{i=1}^{n} C_{i} \varphi_{i}(t, \varepsilon)+\int_{0}^{1} H(t, s, \varepsilon) z(s, \varepsilon) d s,
$$

where

$$
\begin{gathered}
\varphi_{i}(t, \varepsilon)=\int_{0}^{1} \sum_{j=0}^{m_{1}+1} H_{j}(t, x) \Phi_{i}^{(j)}(x, \varepsilon) d x, \quad i=1, \ldots, n, \\
H(t, s, \varepsilon)=\frac{1}{\varepsilon} \int_{s}^{1} \sum_{j=0}^{m_{1}+1} H_{j}(t, x) K^{(j)}(x, s, \varepsilon) d x .
\end{gathered}
$$

Assume that the following condition is valid.

C6) $\lambda=1$ is not an eigenvalue of the kernel $H(t, s, \varepsilon)$.

In view of condition (C6), the integral equation (3.2) has a unique solution [22] that can be represented in the form

$$
z(s, \varepsilon)=\bar{F}(t, \varepsilon)+\sum_{i=1}^{n} C_{i} \bar{\varphi}_{i}(t, \varepsilon)
$$

where

$$
\begin{aligned}
& \bar{F}(t, \varepsilon) \equiv F(t)+\int_{0}^{1} R(t, s, \varepsilon) F(s) d s, \\
& \bar{\varphi}_{i}(t, \varepsilon)=\varphi_{i}(t, \varepsilon)+\int_{0}^{1} R(t, s, \varepsilon) \varphi_{i}(s, \varepsilon) d s, \quad i=1, \ldots, n,
\end{aligned}
$$

and $R(t, s, \varepsilon)$ is a resolvent of the kernel $H(t, s, \varepsilon)$ such that

$$
R(t, s, \varepsilon)=\bar{R}(t, s)+O(\varepsilon),
$$


where $\bar{R}(t, s)$ is a part of $R(t, s, \varepsilon)$ independent of $\varepsilon$. The last representation follows easily by means of (2.9), (3.3) and boundedness of the kernel $H(t, s, \varepsilon)$ as $\varepsilon \rightarrow 0$. Use (3.4) in the right-hand side of (3.1) to obtain the solution of the boundary value problem (2.1) and (2.2) in the form

$$
y(t, \varepsilon)=\sum_{i=1}^{n} C_{i} Q_{i}(t, \varepsilon)+P(t, \varepsilon)
$$

where

$$
\begin{aligned}
Q_{i}(t, \varepsilon) & =\Phi_{i}(t, \varepsilon)+\frac{1}{\varepsilon} \int_{0}^{t} K(t, s, \varepsilon) \bar{\varphi}_{i}(s, \varepsilon) d s, \\
P(t, \varepsilon) & =\frac{1}{\varepsilon} \int_{0}^{t} K(t, s, \varepsilon) \bar{F}(s, \varepsilon) d s,
\end{aligned}
$$

where $\bar{F}(t, \varepsilon)$ and $\bar{\varphi}_{i}(t, \varepsilon)$ are defined in (3.5). Now, we determine the unknown constants $C_{i}, i=1, \ldots, n$, in (3.7) which satisfy the boundary conditions (2.2). Thus, we need to solve the system of algebraic equations

$$
\left\{\begin{array}{l}
C_{1}\left(1+d_{11}(\varepsilon)\right)+C_{2} d_{12}(\varepsilon)+\ldots+C_{n} d_{1 n}(\varepsilon)=a_{1}-e_{1}(\varepsilon) \\
\ldots \ldots \ldots \\
C_{1} d_{n 1}(\varepsilon)+C_{2} d_{n 2}(\varepsilon)+\ldots+C_{n}\left(1+d_{n n}(\varepsilon)=a_{n}-e_{n}(\varepsilon)\right.
\end{array}\right.
$$

where

$$
\begin{aligned}
d_{i k}(\varepsilon) & =\sum_{j=0}^{l_{i}} \frac{\beta_{i j}}{\varepsilon} \int_{0}^{1} K^{(j)}(1, s, \varepsilon) \bar{\varphi}_{k}(s, \varepsilon) d s, \quad k, i=1, \ldots, n, \\
e_{i}(\varepsilon) & =\sum_{j=0}^{l_{i}} \frac{\beta_{i j}}{\varepsilon} \int_{0}^{1} K^{(j)}(1, s, \varepsilon) \bar{F}(s, \varepsilon) d s, \quad i=1, \ldots, n .
\end{aligned}
$$

In view of (2.9), (3.5) and (3.6) we have following asymptotic representations

$$
d_{i k}(\varepsilon)=\overline{d_{i k}}+O(\varepsilon), \quad e_{i}(\varepsilon)=\overline{e_{i}}+O(\varepsilon) \quad i=1, \ldots, n,
$$

where

$$
\bar{d}_{i 1}(\varepsilon)=\sum_{j=0}^{l_{i}} \beta_{i j} \int_{0}^{1} \frac{\bar{W}_{n-1}^{(j)}(1, s)}{\mu(s) \alpha_{1, m_{1}} \bar{W}(s)} \bar{H}_{m_{1}+1}(s, 0) d s, \quad i=1, \ldots, n,
$$




$$
\begin{gathered}
\bar{d}_{i k}(\varepsilon)=\sum_{j=0}^{l_{i}} \beta_{i j} \int_{0}^{1} \frac{\bar{W}_{n-1}^{(j)}(1, s)}{\mu(s) \alpha_{1, m_{1}} \bar{W}(s)} \bar{H}_{k}(s) d s, \quad i=1, \ldots, n, k=2, \ldots, n, \\
\bar{e}_{i}=-\sum_{j=0}^{l_{i}} \beta_{i j} \int_{0}^{1} \frac{\bar{W}_{n-1}^{(j)}(1, s)}{\mu(s) \bar{W}(s)} \bar{F}(s) d s, \quad i=1, \ldots, n,
\end{gathered}
$$

the functions $\bar{H}_{k}(t), \bar{H}_{i}(t, x)$, and $\bar{F}(t)$ have the form

$$
\begin{gathered}
\bar{H}_{k}(t) \equiv(-1)^{k} \frac{\bar{\Delta}_{k}}{\alpha_{1, m_{1}} \bar{\Delta}} \bar{H}_{m_{1}}+1(t, 0)+\int_{0}^{1} \sum_{i=0}^{m_{1}+1} \bar{H}_{i}(t, x) \frac{\bar{\Delta}_{k-1}^{(i)}(x)}{\bar{\Delta}} d x, k=2, \ldots, n, \\
\bar{H}_{i}(t, x) \equiv H_{i}(t, x)+\int_{0}^{1} \bar{R}(t, s) H_{i}(s, x) d s, \quad i=0, \ldots, m_{1}+1, \\
\bar{F}(t) \equiv F(t)+\int_{0}^{1} \bar{R}(t, s) F(s) d s .
\end{gathered}
$$

For the main determinant $\omega(\varepsilon)$ of the system (3.9), we have the following asymptotic representation: $\omega(\varepsilon)=\bar{\omega}+O(\varepsilon)$, where

$$
\bar{\omega}=\left|\begin{array}{cccc}
1+\bar{d}_{11} & \bar{d}_{12} & \ldots & \bar{d}_{1 n} \\
\bar{d}_{21} & 1+\bar{d}_{22} & \ldots & \bar{d}_{2 n} \\
\ldots & \ldots & \ldots & \ldots \\
\bar{d}_{n 1} & \bar{d}_{n 2} & \ldots & 1+\bar{d}_{n n}
\end{array}\right|
$$

Assume now that the following condition is valid:

C7) $\bar{\omega} \neq 0$.

Thus, in view of conditions (C1)-(C7), the singularly perturbed boundary value problem (2.1) and (2.2) has a unique solution $y(t, \varepsilon)$, which can be presented in the following form

$$
y(t, \varepsilon)=\sum_{i=1}^{n} C_{i} Q_{i}(t, \varepsilon)+P(t, \varepsilon),
$$

where $Q_{i}(t, \varepsilon), i=1, \ldots, n$, and $P(t, \varepsilon)$, defined by the formula (3.8),

$C_{i}(\varepsilon), i=1, \ldots, n$, are the solutions of the system (3.9). Then the following Theorem is valid. 
Theorem 1. Under conditions $(C 1)-(C 7)$, for the solution $y(t, \varepsilon)$ of the boundary value problem (2.1) and (2.2), and its derivatives in the interval $0 \leq t \leq 1$ the following asymptotic estimations hold as $\varepsilon \rightarrow 0$

$$
\begin{aligned}
&\left|y^{(j)}(t, \varepsilon)\right| \leq \frac{c}{\bar{\omega}}\left[\frac{1}{\alpha_{1, m_{1}}} \sum_{k=1}^{n}\left|\alpha_{k}\right| \cdot \max _{0 \leq t \leq 1}\left|H_{m_{1}+1}(t, 0)\right|+\sum_{k=2}^{n}\left|\alpha_{k}\right|+\right. \\
&\left.+\sum_{k=1}^{n}\left|\alpha_{k}\right| \cdot \varepsilon^{m_{1}-j} \exp \left(-\gamma \frac{t}{\varepsilon}\right)+\max _{0 \leq t \leq 1}|F(t)|\right] \\
& j=\overline{0, n-1}, \quad 0 \leq t \leq 1,
\end{aligned}
$$

where $C>0, \gamma>0$ are constants independent of $\varepsilon$.

Proof of Theorem 1. By means of (2.9), (2.13), (3.3), (3.5), (3.6) and (3.12), we obtain the following asymptotic representation for functions $Q_{i}(t, \varepsilon), i=1, \ldots, n$, defined in (3.8), as $\varepsilon \rightarrow 0$

$$
\begin{aligned}
& Q_{1}^{(j)}(t, \varepsilon)=\int_{0}^{t} \frac{\bar{W}_{n-1}^{(j)}(t, s)}{\alpha_{1, m_{1}} \mu(s) \bar{W}(s)} \bar{H}_{m_{1}+1}(s, 0) d s+ \\
& +\varepsilon^{m_{1}-j} \frac{y_{n 0}(t) \mu^{j}(t)}{\mu^{m_{1}}(0) \alpha_{1, m_{1}}} \exp \left(\frac{1}{\varepsilon} \int_{0}^{t} \mu(x) d x\right)+ \\
& +O\left(\varepsilon+\varepsilon^{m_{1}+1-j} \exp \left(\frac{1}{\varepsilon} \int_{0}^{t} \mu(x) d x\right)\right), j=0, \ldots, n-2 ; \\
& Q_{1}^{(n-1)}(t, \varepsilon)=\int_{0}^{t} \frac{\bar{W}_{n-1}^{(n-1)}(t, s)}{\alpha_{1, m_{1}} \mu(s) \bar{W}(s)} \bar{H}_{m_{1}+1}(s, 0) d s+ \\
& +\frac{\bar{H}_{m_{1}+1}(t, 0)}{\mu(t) \alpha_{1, m_{1}}}+\frac{1}{\varepsilon^{n-1-m_{1}}} \frac{y_{n 0}(t) \mu^{n-1}(t)}{\mu^{m_{1}}(0) \alpha_{1, m_{1}}} \times \\
& \times \exp \left(\frac{1}{\varepsilon} \int_{0}^{t} \mu(x) d x\right)+O\left(\varepsilon+\frac{1}{\varepsilon^{n-2-m_{1}}} \exp \left(\frac{1}{\varepsilon} \int_{0}^{t} \mu(x) d x\right)\right), \\
& Q_{i}^{(j)}(t, \varepsilon)=\frac{\bar{\Delta}_{i-1}^{(j)}(t)}{\bar{\Delta}}-\int_{0}^{t} \frac{\bar{W}_{n-1}^{(j)}(t, s)}{\mu(s) \bar{W}(s)} \bar{H}_{i}(s) d s+\varepsilon^{m_{1}-j} \frac{(-1)^{i-1} y_{n 0}(t) \mu^{j}(t) \bar{\Delta}_{i}}{\mu^{m_{1}}(0) \alpha_{1, m_{1}} \bar{\Delta}} \times
\end{aligned}
$$




$$
\begin{aligned}
& \times \exp \left(\frac{1}{\varepsilon} \int_{0}^{t} \mu(x) d x\right)+O\left(\varepsilon+\varepsilon^{m_{1}+1-j} \exp \left(\frac{1}{\varepsilon} \int_{0}^{t} \mu(x) d x\right)\right) \\
& Q_{i}^{(n-1)}(t, \varepsilon)=\frac{\bar{\Delta}_{i-1}^{(n-1)}(t)}{\bar{\Delta}}-\int_{0}^{t} \frac{\bar{W}_{n-1}^{(n-1)}(t, s)}{\mu(s) \bar{W}(s)} \bar{H}_{i}(s) d s-\frac{\bar{H}_{i}(t)}{\mu(t)}+ \\
& +\frac{1}{\varepsilon^{n-1-m_{1}}} \frac{(-1)^{i-1} y_{n 0}(t) \mu^{n-1}(t) \bar{\Delta}_{i}}{\mu^{m_{1}}(0) \alpha_{1, m_{1}} \bar{\Delta}} \exp \left(\frac{1}{\varepsilon} \int_{0}^{t} \mu(x) d x\right)+ \\
& +O\left(\varepsilon+\frac{1}{\varepsilon^{n-2-m_{1}}} \exp \left(\frac{1}{\varepsilon} \int_{0}^{t} \mu(x) d x\right), i=2, \ldots, n\right. \\
& P^{(j)}(t, \varepsilon)=-\int_{0}^{t} \frac{W_{n-1}^{(j)}(t, s)}{\mu(s) \bar{W}(s)} \bar{F}(s) d s+O(\varepsilon), j=0, \ldots, n-2, \\
& P^{(n-1)}(t, \varepsilon)=-\int_{0}^{t} \frac{W_{n-1}^{(n-1)}(t, s)}{\mu(s) \bar{W}(s)} \bar{F}(s) d s-\frac{\bar{F}^{\prime}(t)}{\mu(t)}+ \\
& +\frac{y_{n 0}(t) \mu^{n-1}(t) \bar{F}(0)}{\mu^{n}(0)} \exp \left(\frac{1}{\varepsilon} \int_{0}^{t} \mu(x) d x\right)+O(\varepsilon)
\end{aligned}
$$

In view of (3.10), $C_{i}(\varepsilon)$ can be asymptotically represented in the form

$$
C_{i}(\varepsilon)=\frac{\overline{\omega_{i}}}{\bar{\omega}}+O(\varepsilon), \quad i=1, \ldots, n,
$$

where $\overline{\omega_{i}}$ is the determinant obtained from $\bar{\omega}$ by substituting the $i$-th column by $\left(\begin{array}{c}a_{1}-\bar{e}_{1} \\ \ldots \\ a_{n}-\bar{e}_{n}\end{array}\right)$. By using presentations of (3.16), (3.15) and condition (C2), we derive the asymptotic estimations (3.14) from (3.13). The Theorem is proved.

Definition 3. The solution of singularly perturbed integro-differential equation at the point $t=t_{0}$ has the phenomenon of the $m$-th order initial jump, if at the point $t=t_{0}$ the solution of boundary value problem (2.1) and (2.2) have the pole of $\varepsilon$ :

$$
y^{(j)}\left(t_{0}, \varepsilon\right)=O(1), j=\overline{0, m}, \quad y^{(m+j)}\left(t_{0}, \varepsilon\right)=O\left(\frac{1}{\varepsilon^{j}}\right), j=\overline{1, n-1-m}, \varepsilon \rightarrow 0 .
$$


By Theorem 1, we have that

$$
y^{(j)}(0, \varepsilon)=O(1), j=\overline{0, m_{1}}, y^{\left(m_{1}+j\right)}(0, \varepsilon)=O\left(\frac{1}{\varepsilon^{j}}\right), j=\overline{1, n-1-m_{1}}, \varepsilon \rightarrow 0 .
$$

Hence, according to Definition 3 one can easily see that the solution of the problem (2.1) and (2.2) has the $m_{1}$-th order initial jump at point $t=0$.

Consider the modified degenerate integro-differential equation:

$$
\begin{aligned}
L_{0} \bar{y} & \equiv A_{1}(t) \bar{y}^{(n-1)}(t)+\sum_{i=2}^{n} A_{i}(t) \bar{y}^{(n-i)}(t) \\
& =F(t)+\int_{0}^{1} \sum_{i=0}^{m_{1}+1} H_{i}(t, x) \bar{y}^{(i)}(x) d x+\Delta(t),
\end{aligned}
$$

with boundary conditions

$$
\begin{aligned}
h_{1} \bar{y}(t) & \equiv \sum_{j=0}^{m_{1}} \alpha_{1 j} \bar{y}^{(j)}(0)+\sum_{j=0}^{l_{1}} \beta_{1 j} \bar{y}^{(j)}(1)=a_{1}-\alpha_{1, m_{1}} \Delta_{0}, \\
h_{i} \bar{y}(t) & \equiv \sum_{j=0}^{m_{i}} \alpha_{i j} \bar{y}^{(j)}(0)+\sum_{j=0}^{l_{i}} \beta_{i j} \bar{y}^{(j)}(1)=a_{i}, \quad i=\overline{2, n}
\end{aligned}
$$

where

$$
\Delta(t)=\Delta_{0} H_{m_{1}+1}(t, 0) .
$$

It is seen that, the modified degenerate problem (3.17) and (3.18) has additional terms $\Delta(t)$ and $\Delta_{0}$, where $\Delta(t)$ is called the initial jump of the integral term and $\Delta_{0}$ is called the initial jump of the $m_{1}$-th order derivative of the solution. The following condition is needed in the paper.

C8) The modified degenerate problem (3.17) and (3.18) has a unique solution $\bar{y}(t)$.

Theorem 2. If conditions (C1)-(C8) and (3.19) are satisfied, then in the interval $0 \leq t \leq 1$ the following asymptotic estimation is valid as $\varepsilon \rightarrow 0$

$$
\left|y^{(j)}(t, \varepsilon)-\bar{y}^{(j)}(t)\right| \leq C\left[\varepsilon+\varepsilon^{m_{1}-j} \exp \left(-\gamma_{\varepsilon}^{t}\right)\right], j=0, \ldots, n-1,
$$

where $C>0, \gamma>0$ are constants independent of $\varepsilon$.

Proof of Theorem 2. Let $u(t, \varepsilon)=y(t, \varepsilon)-\bar{y}(t)$ where $y(t, \varepsilon)$ is the solution of the problem (2.1) and (2.2) and $\bar{y}(t)$ is the solution of the problem (3.17) and (3.18). We substitute the variable $y(t, \varepsilon)=u(t, \varepsilon)+\bar{y}(t)$ to obtain the following problem 


$$
\begin{aligned}
L_{\varepsilon} u & =\int_{0}^{1} \sum_{i=0}^{m_{1}+1} H_{i}(t, x) u^{(i)}(x, \varepsilon) d x-\varepsilon \bar{y}^{(n)}-\Delta(t), \\
h_{1} u(t, \varepsilon) & =\alpha_{1, m_{1}} \Delta_{0}, h_{i} u(t, \varepsilon)=0, i=2, \ldots, n .
\end{aligned}
$$

The problem (3.21) is of the same type as the problem (2.1) and (2.2). By applying Theorem 1 and estimates (3.14), we obtain the following asymptotic estimation for the solution $u(t, \varepsilon)$ of the problem (3.21) as $\varepsilon \rightarrow 0$

$$
\begin{aligned}
\left|u^{(j)}(t, \varepsilon)\right| \leq & C\left[\varepsilon+\max _{0 \leq t \leq 1}\left|\Delta(t)-\Delta_{0} H_{m_{1}+1}(t, 0)\right|+\varepsilon^{m_{1}-j} \exp \left(-\gamma \frac{t}{\varepsilon}\right)\right], \\
& j=0, \ldots, n-1 .
\end{aligned}
$$

From (3.22) and (3.19) one can obtain (3.20). The Theorem is proved.

According to (3.22), it is true that

$$
\begin{aligned}
\lim _{\varepsilon \rightarrow 0} y^{(j)}(t, \varepsilon) & =\bar{y}^{(j)}(t), j=\overline{0, m_{1}-1}, 0 \leq t \leq 1, \\
\lim _{\varepsilon \rightarrow 0} y^{\left(m_{1}+j\right)}(t, \varepsilon) & =\bar{y}^{\left(m_{1}+j\right)}(t), j=\overline{0, n-1-m_{1}}, 0<t \leq 1 .
\end{aligned}
$$

From (3.23) it follows that the solution $y(t, \varepsilon)$ of the boundary value problem (2.1) and (2.2) converges to the solution $\bar{y}(t)$ of the modified degenerate problem (3.17) and (3.18) as $\varepsilon \rightarrow 0$. We note that the limits for $y^{\left(m_{1}+j\right)}, j=\overline{0, n-1-m_{1}}$ of (3.23) are not uniform on the interval $0 \leq t \leq 1$. They are uniform an interval $0<t_{0} \leq t \leq 1$ , where $t_{0}$ is sufficiently small but fixed number as $\varepsilon \rightarrow 0$.

\section{EXAMPLE}

Let us consider the following singularly perturbed boundary value problem

$$
\begin{aligned}
\varepsilon y^{\prime \prime \prime}+a y^{\prime \prime} & =b+\int_{0}^{1} \delta y^{\prime \prime}(x, \varepsilon) d x, a>0, \delta \neq 0, \delta \neq a, 0<t<1, \\
h_{1} y(t, \varepsilon) & \equiv y(0, \varepsilon)+y^{\prime}(0, \varepsilon)=a_{1}, \\
h_{2} y(t, \varepsilon) & \equiv y(0, \varepsilon)=a_{2}, \\
h_{3} y(t, \varepsilon) & \equiv y(1, \varepsilon)-y^{\prime}(1, \varepsilon)=a_{3},
\end{aligned}
$$

where $a, b, \delta, a_{i}, i=1,2,3$, are known constants. The exact solution of the problem (4.1) has the form 


$$
\begin{aligned}
y(t, \varepsilon)= & a_{2}-\varepsilon \frac{2\left(a_{2}-a_{3}\right)(a-\delta)-b}{a \delta+2(a-\delta) \varepsilon-\left[2 a^{2}-a \delta+2(a-\delta) \varepsilon\right] e^{-\frac{a}{\varepsilon}}}+ \\
& +\left(a_{1}-a_{2}+\frac{2 a\left(a_{2}-a_{3}\right)(a-\delta)-a b}{a \delta+2(a-\delta) \varepsilon-\left[2 a^{2}-a \delta+2(a-\delta) \varepsilon\right] e^{-\frac{a}{\varepsilon}}}\right) t+ \\
& +\frac{a \delta\left(a_{2}-a_{3}\right)+b \varepsilon-\left[a b-\delta a\left(a_{3}-a_{2}\right)+b \varepsilon\right] e^{-\frac{a}{\varepsilon}}}{a \delta+2(a-\delta) \varepsilon-\left(2 a^{2}-a \delta+2(a-\delta) \varepsilon\right) e^{-\frac{a}{\varepsilon}}} t^{2}+ \\
& +\varepsilon \frac{2\left(a_{2}-a_{3}\right)(a-\delta)-b}{a \delta+2(a-\delta) \varepsilon-\left[2 a^{2}-a \delta+2(a-\delta) \varepsilon\right] e^{-\frac{a}{\varepsilon}}} e^{-\frac{a t}{\varepsilon}} .
\end{aligned}
$$

It is seen that $y^{(i)}(0, \varepsilon)=O(1), y^{\prime \prime}(0, \varepsilon)=O\left(\frac{1}{\varepsilon}\right), i=0,1$, as $\varepsilon \rightarrow 0$. That is, the solution of problem (4.1) has the first order initial jump at $\mathrm{t}=0$. Next, one can obtain that

$$
\lim _{\varepsilon \rightarrow 0} y(t, \varepsilon)=\bar{y}(t), o \leq t \leq 1, \lim _{\varepsilon \rightarrow 0} y^{(j)}(t, \varepsilon)=\bar{y}^{(j)}(t), i=1,2, o<t \leq 1,
$$

where $\bar{y}(t)=a_{2}+\left(a_{1}-a_{2}+\frac{2\left(a_{2}-a_{3}\right)(a-\delta)+b}{\delta}\right) t+\left(a_{2}-a_{3}\right) t^{2}$ is the solution of the modified degenerated problem

$$
\begin{aligned}
a \bar{y}^{\prime \prime} & =b+\int_{0}^{1} \delta \bar{y}^{\prime \prime}(x) d x+\Delta(t), a>0, \delta \neq 0, \delta \neq a, 0<t<1, \\
h_{1} \bar{y}(t) & \equiv \bar{y}(0)+\bar{y}^{\prime}(0)=a_{1}+\Delta_{0}, h_{2} \bar{y}(t) \equiv \bar{y}(0)=a_{2}, \\
h_{3} \bar{y}(t) & \equiv \bar{y}(1)-\bar{y}^{\prime}(1)=a_{3},
\end{aligned}
$$

where

$$
\Delta(t)=\delta \Delta_{0} .
$$

By applying (4.4) to (4.3), we obtain the initial jump of the first order derivative $\Delta_{0}=\frac{2(a-\delta)\left(a_{2}-a_{3}\right)-b}{\delta}$, and then from (4.4) the initial jump of integral part $\Delta(t)=$ $2(a-\delta)\left(a_{2}-a_{3}\right)-b$.

\section{CONCLUSiON}

The method of initial jumps has been applied to the singular linear integro-differential equation. The modified degenerated equation with the initial jump of the integral term is constructed such that the solution of the original singular problem converges to the solution of the modified equation with the jumps in the initial values. It is verified that the order of the jump derivative depends on the highest order of derivatives in the right-hand side of the original equation. 


\section{REFERENCES}

[1] N. Bogoliubov and Y. A. Mitropolskii, Asymptotic methods in the theory of nonlinear oscillations. Delhi: Hindustan Publ. Corp., 1961.

[2] W. Eckhaus, Matched asymptotic expansions and singular perturbations. Amsterdam, London: North-Holland, 1973.

[3] M. Fedoryuk, Asymptotic methods for linear ordinary differential equations (In Russian.). Moscow: Nauka, 1983.

[4] J. Grasman, Asymptotic methods for relaxation oscillations and applications. Berlin, Heidelberg, New York: Springer-Verlag, 1987.

[5] A. Kapila, Asymptotic treatment of chemically reacting systems. Boston, London, Melbourne: Pitman, 1983.

[6] K. A. Kasymov, "The initial jump problem for non-linear systems of differential equations containing a small parameter (in russian)," Doklady Akademii Nauk USSR, vol. 179, no. 2, pp. 275$278,1968$.

[7] K. A. Kasymov, "Asymptotics of the solutions of the problem with an initial jump for a system of differential equations of hyperbolic type with a small parameter at the derivative (in russian)," Doklady Akademii Nauk USSR, vol. 196, no. 2, pp. 274-277, 1971.

[8] J. Kevorkian and J. D. Cole, Singular perturbation methods in applied mathematics. Berlin, Heidelberg, New York: Springer-Verlag, 1981.

[9] P. Lagerstrom, Matched asymptotic expansions. Berlin, Heidelberg, New York: Springer-Verlag, 1988.

[10] S. Lomov, Introduction to the general theory of singular perturbations. Providence, RI: American Mathematical Society, 1992.

[11] E. Mishchenko and N. Rozov, Differential equations with small parameter and relaxation oscillations. New York: Plenum Press, 1980.

[12] D. Nurgabyl, "Asymptotic estimates of the solution of a restoration problem with an initial jump," Journal of Applied Mathematics, vol. 2014, p. Article ID 956402, 2014.

[13] F. Olver, Introduction to asymptotics and special functions. New York, London: Academic Press, 1974.

[14] R. O'Malley, Introduction to singular perturbations. New York: Academic Press, 1974.

[15] R. O'Malley, Singular perturbations methods for ordinary differential equations. Berlin, Heidelberg, New York: Springer-Verlag, 1991.

[16] A. Ronto and M. Ronto, "On the investigation of some boundary value problems with nonlinear conditions," Math. Notes, Miskolc, vol. 1, no. 1, pp. 43-55, 2000.

[17] M. Ronto and K. Marynets, "On the parametrization of boundary-value problems with three-point non-linear restrictions," Math. Notes, Miskolc, vol. 13, no. 1, pp. 91-106, 2012.

[18] M. Ronto and A. Samoilenko, Numerical-analitic methods in the theory of boundary-value problems. Singapore: World Scientific, 2000.

[19] J. A. Sanders, F. Verhulst, and J. Murdock, Averaging methods in nonlinear dynamical systems. Berlin, Heidelberg, New York: Springer-Verlag, 1985.

[20] A. Vasil'eva, "On the development of singular perturbation theory at moscow state university and elsewhere," SIAM Review, vol. 36, pp. 440-452, 1994, doi: 10.1137/1036100.

[21] A. Vasil'eva and V. Butuzov, Singularly perturbed differential equations of parabolic type, ser. Asymptotic Analysis. Berlin: Springer-Verlag, 1983, vol. II.

[22] A. Vasil'eva, V. Butuzov, and L. Kalachev, The boundary function method for singular perturbation problems, ser. SIAM Studies in Applied Mathematics. Philadeplhia: SIAM, 1995, vol. 14, doi: $10.1137 / 1.9781611970784$. 
[23] I. Vishik and L. A. Lyusternik, "On the initial jump for non-linear differential equations containing a small parameter (in russian)," Doklady Akademii Nauk USSR, vol. 132, no. 6, pp. 1242-1245, 1960.

[24] M. Vishik and L. Lyusternik, "Regular degeneration and boundary layer for linear differential equations with small parameter multiplying the highest derivatives," Amer. Math. Soc. Transl., vol. 20, no. 2, pp. 239-364, 1962.

Authors' addresses

M.K. Dauylbayev

Al-Farabi Kazakh National University, Department of Mechanics and Mathematics, al-Farabi 71, 050040 Almaty, Kazakhstan

E-mail address: dmk57@mail.com

\section{N. Atakhan}

Al-Farabi Kazakh National University, Department of differential equations and control theory, Faculty of Mechanics and Mathematics, 050040 Almaty, Kazakhstan

E-mail address: atakhan-nilupar@mail.ru 